\title{
The Incidence of Central Retinal Artery Occlusion in Olmsted County, Minnesota
}

\author{
Jacqueline A. Leavitt ${ }^{1}$, Theresa A. Larson ${ }^{1}$, David O. Hodge ${ }^{2}$, and Rachel E. Gullerud ${ }^{2}$ \\ ${ }^{1}$ Department of Ophthalmology, Mayo Clinic, Rochester, MN \\ ${ }^{2}$ Department of Health Sciences Research, Mayo Clinic, Rochester, MN
}

\section{Abstract}

Purpose-To determine the incidence of central retinal artery occlusion in Olmsted County, Minnesota.

Design-Retrospective chart review.

Methods-Medical records of all patients living in Olmsted County, MN between 1976 and 2005 diagnosed with central retinal artery occlusion cases were identified using the Rochester Epidemiology Project medical records linkage system.

Results-Forty-three cases were identified for an unadjusted annual incidence in females of 1.02 per 100,000 and in males 1.67 per 100,000 with a combined incidence of 1.33 . Incidence rates were also age- and/or sex-adjusted to the 2000 census figures for the U.S. white population using direct standardization. Age adjusted annual incidence per 100,000 for females was 1.15 (95\% confidence interval [CI], $0.60-1.71$ ), for males was 2.78 (95\% CI, $1.69-3.86$ ), and combined was $1.87(95 \% \mathrm{CI}, 1.31-2.43)$. When adjusted for age and sex, the incidence was 1.90 per $100,000$ (95\% CI, $1.33-2.47)$.

Conclusion-Central retinal artery occlusion is a rare event. The incidence is 1.3 per 100,000 in Olmsted County, Minnesota, or 1.90 per 100,000 when age- and sex-adjusted for the United States white population.

\section{Introduction}

The classic clinical presentation of central retinal artery occlusion (CRAO) has changed very little since it was first described by Von Graefe in 1859. Clinically, patients present with profound vision loss from ischemia to the inner retinal layer and an afferent pupillary defect. Vision ranges from light perception to counting fingers in the majority of patients.

\footnotetext{
(C) 2011 Elsevier Inc. All rights reserved. 507-284-4612; leavitt.jacqueline@mayo.edu.

The authors have no financial or conflicting interests to disclose.

Author contributions:

1. Design and conduct of the study (JL, TL)

2. Collection, management, analysis and interpretation of the data (JL, TL, DH, RG)

3. Preparation, review or approval of the manuscript. (JL, TL, DH, RG)
}

Inquiries to Jacqueline A. Leavitt, Mayo Clinic Rochester, 200 First St. SW, Rochester, MN 55905; phone 507-284-3726; fax

Publisher's Disclaimer: This is a PDF file of an unedited manuscript that has been accepted for publication. As a service to our customers we are providing this early version of the manuscript. The manuscript will undergo copyediting, typesetting, and review of the resulting proof before it is published in its final citable form. Please note that during the production process errors may be discovered which could affect the content, and all legal disclaimers that apply to the journal pertain. 
On fundus exam, the posterior pole is opacified except for a cherry red spot where the nerve fiber layer is the thinnest. There may be attenuation of the retinal arterioles and "boxcarring" or segmentation of the blood column. In $14-26 \%$ of patients, there is cilioretinal artery sparing 2,3 which has important implications for visual prognosis depending on how much of the papillomacular bundle is spared. With sparing of the foveola, $80 \%$ improve to $20 / 50$ vision or better.

Voluminous literature has been written about CRAO, but only a few studies have reported on incidence. Karjalainen in Helsinki reported an increasing incidence of retinal artery occlusions per 1000 "first out-patient department visits" over the time studied (1959-1969) starting at approximately $0.5 / 1000$ and ending at approximately $1 / 1000 .{ }^{4}$ At Wills Eye Hospital, CRAO was estimated to occur in 1 per 10,000 outpatient visits. ${ }^{5}$ In Israel, it was estimated to occur in 0.85 per 100,000 per year or 1.13 per 10,000 outpatient visits. ${ }^{6}$ In the district of Split, Croatia, it was estimated to occur as an annual incidence of 0.7 (range 0.21.7) per 100,000 population with an annual frequency of 3.4 over a 16 year period. ${ }^{7}$ In this study as in most others, men were more frequently affected and the majority of patients were in their 60s. The clinical characteristics and outcomes of the patients were not described.

In this study we determined the incidence of CRAO in Olmsted County, Minnesota and describe the clinical characteristics of patients who have this disease.

\section{Methods}

Patient charts that indicated a diagnosis of 'retinal artery occlusion' from January 1, 1976, through December 31, 2005 were selected and reviewed using the Rochester Epidemiology Project. The Rochester Epidemiology Project is a medical records linkage system established to study the epidemiology of disease for residents of Olmsted County in Southeastern Minnesota. It links and indexes diagnostic and procedure information from Mayo Clinic in Rochester, Minnesota and other facilities that provide health care to residents in this community (Olmsted Community Hospital, Olmsted Medical Group, the University of Minnesota and Department of Veterans Affairs hospitals in Minneapolis). ${ }^{8}$

Records were identified through the Rochester Epidemiology Project based the hospital adaptation of the International Classification of Diseases and International Classification of Diseases-9 codes for: retinal embolism, retinal infarction, Hollenhorst plaque, retinal ischemia, retinal artery occlusion, retinal artery obstruction, artery eye occlusion, retinal vascular occlusion, unspecified retinal ischemia, partial retinal arterial occlusion, retinal arterial branch occlusion and transient retinal arterial occlusion. Patients were eligible for including in this study if their record indicated a clinical history of abrupt vision loss and fundus findings of retinal opacification or a cherry red spot or both. Cases were excluded if there was a recent invasive or surgical procedure (including ocular surgery) known to precipitate a CRAO. Also excluded were cases with an ocular condition that prevented a view of the fundus.

Yearly incidence rates for each age and sex group were determined by dividing the number of cases within each group by the estimated total Olmsted County resident population for that year. Population figures for 1990 and 2000 came from US census, and populations for the inter-census years were estimated by using linear interpolation. Estimates from the State of Minnesota Demographer's Office were used to aid with linear interpolation between census years. Incidence rates were also age and sex-adjusted to the 2000 census figures for the U.S. white population. The $95 \%$ confidence intervals (CI) were calculated by assuming a Poisson distribution. Mortality was estimated by using the Kaplan-Meier method. 
The racial distribution of Olmsted County residents in 1990 was 95.7\% Caucasian, 3.0\% Asian-American, $0.7 \%$ African-American, $0.3 \%$ each Native American and other. The population of this county $(106,470$ in 1990) is relatively isolated from other urban areas, and virtually all medical care is provided to residents by the Mayo Clinic or the Olmsted Medical Group and their affiliated hospitals. Residency of patients at the time of diagnosis was checked by using information from city and county directories. Patients not living in Olmsted County at the time of their diagnosis were excluded.

\section{Results}

Between 1976 and 2005, out of 740 charts reviewed 43 cases of CRAO were identified using the inclusion and exclusion criteria; $26(60 \%)$ were male and $17(40 \%)$ were female. The mean age at presentation was 74.3 years (range 46-90). Racial characteristics of the patients reflects Olmsted County's population with 93\% Caucasian and 2\% each Asian, African-American and Hispanic. Twenty-seven patients were afflicted in the right eye, 15 patients in the left eye, and one presented with bilateral disease. Patients presented on average within 4 days (0.04 - 30 days) of onset, and 32 (74\%) presented within one or two days. Initial visual acuity was worse than $20 / 400$ in 37 (86\%) patients. Vision ranged from no light perception (14\%) to 20/25 (2\%). The majority of patients (28 or $65 \%$ ) had no prior ocular history. On initial examination, 14 patients (33\%) had retinal arterial emboli and 4 (9\%) had cilioretinal sparing. Cardiovascular risk factors included: hypertension (88\%), diabetes mellitus (21\%), hyperlipidemia (40\%), and smoking $16 \%$. Twenty-four patients (56\%) had multiple cardiovascular risk factors. On presentation, 27 patients $(56 \%)$ were not on anti-coagulation therapy. Workups included transthoracic or transesophageal echocardiogram (TTE, TEE), carotid ultrasound, cerebral angiography or magnetic resonance angiography and erythrocyte sedimentation rate (ESR). Twenty-six patients were examined by TTE or TEE. Valve disease was the most frequent abnormality found (10 patients). A thrombus was found in one patient. Vascular procedures including: carotid ultrasound, cerebral angiography or magnetic resonance angiography were performed 32 times among 26 patients. There was a significant vascular stenosis $(>50 \%)$ in six patients. Thirty-eight patients had a recorded ESR, with a median of 11 (range 0-86). The ESR of 86 was recorded in a patient with known rheumatoid arthritis who subsequently had a negative temporal artery biopsy. Of the 43 CRAO cases, three patients underwent surgical intervention for systemic abnormalities found during the workup for CRAO (one each: bilateral carotid endarterectomy, pacemaker placement, superficial temporal artery to middle cerebral artery bypass). Multiple treatments for CRAO were attempted (ocular massage $(40 \%)$, glaucoma medications (33\%), paracentesis (16\%), anticoagulation (14\%), supraorbital artery cannulation with infusion of heparin (14\%), hyperbaric oxygen (2\%)) although no treatment was superior to others. The average follow up after diagnosis was 5 years (range 2 days -22.5 years). Thirty-five patients were deceased at the conclusion of the study period. Causes of death included coronary events in 14 (33\%), stroke in $5(12 \%)$, and other causes in 15 (35\%). One death was from an unknown cause. The unadjusted annual incidence of CRAO in Olmsted County for females and males was 1.02 and 1.67 per 100,000 , respectively, and the combined incidence was 1.33 per 100,000. The age adjusted annual incidence per 100,000 for females was $1.15(95 \% \mathrm{CI}, 0.60-1.71)$, for males was 2.78 (95\% CI, $1.69-3.86)$, and combined was 1.87 (95\% CI, $1.31-2.43)$. When adjusted for age and sex, the annual incidence was 1.90 per 100,000 (95\% CI, $1.33-2.47$ ).

\section{Discussion}

The age and sex adjusted incidence of central retinal artery occlusion for this sample, and the U.S. white population, is slightly higher at 1.90 per 100,000 than the 0.7 per 100,000 in the district of Split Croatia. The mean age in this study was more than a decade older than it 
was in the Croatian study (74 versus 61.9) with the highest incidence in the $75-84$ year age group compared to the $60-69$ year age group in the Croatian study. ${ }^{7}$ The majority of CRAO cases occurred in males in both studies. When specified, more right eyes occurred in our study and several previous studies $4,7,12$ more left eyes occurred in two studies ${ }^{6,16}$ and right and left eyes were almost equally affected one study. ${ }^{2}$

Fundus findings on initial examination in this study contrast with Hayreh ${ }^{9}$ who found more frequent optic disc pallor, disc edema and cilioretinal sparing and less frequent boxcarring and retinal arteriole attenuation. Differences between our study and that of Hayreh may be related to differences in study design and sample size; Hayreh examined 240 consecutive patients with the explicit purpose of describing the fundus findings whereas our study was retrospective and involved review of clinical records from multiple different clinicians.

CRAO is associated with significant systemic pathologies, often with similar pathogenic mechanisms. The majority of CRAOs are secondary to intraluminal thrombosis or embolism. ${ }^{10}$ Cardiovascular risk factors that predispose to CRAO include hypertension, diabetes, and carotid atherosclerotic disease. Thrombophilic risk factors found to be independent risk factors for CRAO are hypercholesterolemia, smoking, hyperhomocysteinemia and lipoprotein (a) greater than $300 \mathrm{mg} / .{ }^{11}$ Retinal emboli are found in approximately $20 \%$ of central retinal artery occlusions, and portend a poor prognosis and decreased overall survival. ${ }^{12}$ Patients with CRAO have higher rates of significant carotid atherosclerotic disease, cardiovascular events, diabetes mellitus and stroke. ${ }^{12-15}$

Hypertension was most frequent followed by hyperlipidemia and diabetes. The results in this study parallel Duker ${ }^{10}$ and Brown et al. ${ }^{16}$ in which about $60 \%$ of patients had hypertension and $25 \%$ had diabetes.

Other systemic factors associated with CRAO included carotid stenosis, cardiac valvular disease, and giant cell arteritis. Brown et al. noted that carotid stenosis was present in 32\% of patients and Appen et al. ${ }^{12}$ found that it was present in $11 \%$. In this study significant (>50\% occlusion) carotid stenosis was present in $14 \%$ of CRAO patients, and stenosis with mild to moderate plaque in 30\%. Cardiac valve disease was present $23 \%$ of patients in our study, similar to $28 \%$ found in a prior study by Sharma. ${ }^{15}$ Generally, approximately $5 \%$ of retinal arterial occlusions are found secondary to giant cell arteritis while in our study no cases were identified.

Visual outcome after treatment in patients with CRAO was poor, consistent with previous studies. Multiple treatments, including supraorbital cannulation, anticoagulation, and intraocular pressure lowering maneuvers, were provided with no better outcome compared to the patients who received no treatment (28\%). At final follow up, $86 \%$ of eyes had vision of counting fingers or worse. Vision improved in $30 \%$ of patients presenting with counting fingers vision or worse. Similarly, Hayreh et al. found that $22 \%$ of nonarteritic CRAOs with vision of counting fingers or worse improved. ${ }^{17}$ Our slightly higher improvement in vision may be secondary to cilioretinal sparing not documented at the time of the exam.

This study is limited by its retrospective design. Cases may not have been captured if they were inaccurately diagnosed, coded, entered in the chart or if patients failed to present at an Olmsted County facility. Also, the study was based on a United States white population, which may not reflect the overall demographic composition of the United States or other populations around the world. As well, the number of cases identified was so small that epidemiological trends cannot be determined from the data. Our data may have been slightly biased by the possible inclusion of ophthalmic artery occlusions, because most cases were not verified by fluorescein angiography. 
In summary the age- and sex adjusted- annual incidence of CRAO is 1.90 per 100,000. Like previous studies visual outcome is poor and there was no proven benefit for any treatment.

\section{Acknowledgments}

Funding/support: Supported in part by an unrestricted grant to the Department of Ophthalmology from Research to Prevent Blindness, Inc., New York, NY and made possible by the Rochester Epidemiology Project (Grant \#R01AR30582 from the National Institute of Arthritis and Musculoskeletal and Skin Diseases).

\section{References}

1. VonGraefe A. Über embolie der arteria centralis retinae als ursache plötzlicher erblindung. Graefes Arch Ophthalmol. 1859; 5:136-185.

2. Hayreh SS, Zimmerman MB. Central retinal artery occlusion. Visual outcome Am J Ophthalmol. 2005; 140(3):376-391.

3. Brown GC, Shields JA. Cilioretinal arteries and retinal arterial occlusion. Arch Ophthalmol. 1979; 97(1):84-92. [PubMed: 758898]

4. Karjalainen K. Occlusion of the central retinal artery and retinal branch arterioles. Acta Ophthalmol Suppl. 1971; 109:1-95. [PubMed: 4334005]

5. Brown, GC. Retinal arterial obstructive disease. Ryan, SJ., editor. Retina, St. Louis: Mosby; 1994. p. 1361

6. Rumelt S, Dorenboim Y, Rehany U. Aggressive systematic treatment for central retinal artery occlusion. Am J Ophthalmol. 1999; 128(6):733-738. [PubMed: 10612510]

7. Ivanišević M, Karelović D. The incidence of central retinal artery occlusion in the district of Split, Croatia. Ophthalmologica. 2001; 215(3):245-246. [PubMed: 11340401]

8. Kokmen E, Beard CM, O’Brien PC, Kurland LT. Mayo Clin Proc. 1996; 71(3):275-282. [PubMed: 8594286]

9. Hayreh SS, Zimmerman MB. Fundus changes in central retinal artery occlusion. Retina. 2007; 27(3):276-289. [PubMed: 17460582]

10. Duker, JS. Retinal artery occlusion. In: Yanoff, M., et al., editors. Ophthalmology. St. Louis: Mosby; 2004. p. 854

11. Marcucci R, Sodi A, Biambene B, et al. Cardiovascular and thrombophilic risk factors in patients with retinal artery occlusion. Blood Coagul Fibrinolysis. 2007; 18(4):321-326. [PubMed: 17473572]

12. Appen RE, Wray SH, Cogan DS. Central retinal artery occlusion. Am J Ophthalmol. 1975; 79(3): 374-381. [PubMed: 1121994]

13. Savino PJ, Glaser JS, Cassady J. Retinal stroke. Arch Ophthalmol. 1977; 95(7):1185-1189. [PubMed: 880077]

14. Hankey GJ, Slattery JM, Warlow CP. Prognosis and prognostic factors of retinal infarction: a prospective cohort study. BMJ. 1991; 302(6775):499-504. [PubMed: 2012845]

15. Sharma $\mathrm{S}$. The systemic evaluation of acute retinal artery occlusion. Curr Opinion Ophthalmol. 1998; 9(III):1-5.

16. Brown GC, Magargal LE. Central retinal artery obstruction and visual acuity. Ophthalmology. 1982; 89(1):14-19. [PubMed: 7070767]

17. Hayreh SS, Podhajsky PA, Zimmerman MB. Retinal artery occlusion. Associated systemic and ophthalmic abnormalities. Ophthalmology. 2009; 116(10):1928-1936. [PubMed: 19577305] 


\section{Biographies}

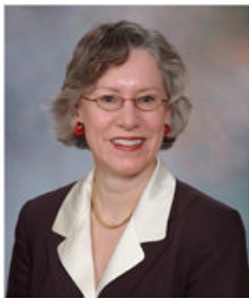

Jacqueline A. Leavitt, M.D.

Undergraduate Mount Holyoke College, Medical school Wayne State University, Ophthalmology residency University of Rochester, Neuro-ophthalmology fellowship Columbia-Presbyterian Medical Center. Currently at Mayo Clinic Rochester.

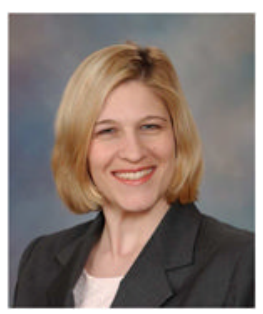

Theresa Larson, MD, graduated from Vanderbilt University School of Medicine in Nashville, TN, in 2005 and completed a residency in ophthalmology at Mayo Clinic, Rochester, MN. She is currently completing a fellowship in ocular immunology at the National Eye Institute, Bethesda, MD. 